\title{
Gauge-invariant decomposition of meson energy in two-dimensional QCD
}

\author{
Yu Jia, ${ }^{1,2, *}$ Rui Yu, ${ }^{1,2, \dagger}$ and Xiaonu Xiong ${ }^{3, \$}$ \\ ${ }^{1}$ Institute of High Energy Physics, Chinese Academy of Sciences, Beijing 100049, China \\ ${ }^{2}$ School of Physics, University of Chinese Academy of Sciences, Beijing 100049, China \\ ${ }^{3}$ Institute for Advanced Simulation, Institut für Kernphysik and Jülich Center for Hadron Physics, \\ Forschungszentrum Jülich, D-52425 Jülich, Germany
}

(Received 6 August 2018; published 25 October 2018)

\begin{abstract}
This work is driven by our curiosity about some basic questions in QCD: how is the energy of a moving hadron partitioned among different gauge-invariant sectors in the QCD Hamiltonian? How is the energy of a massless pion separated between quark and gluon sectors, particularly in the soft pion limit? Is it possible to decompose the celebrated Gell-Mann-Oakes-Renner (GOR) relation? To what extent can we justify the quark potential model from the field-theoretical mass decomposition for heavy quarkonium? Due to the limitations of contemporary nonperturbative tools, we do not yet know answers to these questions in realistic QCD. In this work, we take the 't Hooft model (two-dimensional QCD in the large- $N$ limit) as a prototype that mimics some essential aspects of the true QCD. We investigate the gauge-invariant energy decomposition of a flavor-neutral meson that can carry an arbitrary momentum (including the stationary case), with the meson species ranging from the massless pion to bottomonium. All the aforementioned questions can be addressed satisfactorily within this model; in particular, some unexpected patterns related to the pion are discovered for the first time in two-dimensional QCD. We hope that our study can offer some useful clues and stimulation for future investigation of hadron energy decomposition in realistic QCD.
\end{abstract}

DOI: $10.1103 /$ PhysRevD.98.074024

\section{INTRODUCTION}

It has long been of interest to understand the quark-gluon structure of hadrons directly from the first principle of QCD, especially to unravel how the mass of a hadron is partitioned among the expectation values of different sectors of the QCD Hamiltonian. Obviously, such a decomposition calls for a fully nonperturbative treatment. The early work in this direction was pioneered by $\mathrm{Ji}[1,2]$, who carried out a gauge-invariant decomposition of nucleon mass, with estimates based on some phenomenological inputs. For the first time from the angle of lattice QCD, some exploratory studies were recently published concerning mass decomposition for several species of mesons, including the pion, $\rho$, and lowestlying charmonia [3].

In spite of these existing studies, there remain many basic questions in $\mathrm{QCD}_{4}$ that are inaccessible to current nonperturbative techniques. Such open questions include the following: How is the energy of a moving hadron

\footnotetext{
*jiay@ihep.ac.cn

yurui@ihep.ac.cn

¥x.xiong@fz-juelich.de
}

Published by the American Physical Society under the terms of the Creative Commons Attribution 4.0 International license. Further distribution of this work must maintain attribution to the author(s) and the published article's title, journal citation, and DOI. Funded by SCOAP. partitioned among different gauge-invariant sectors? Can we have some concrete knowledge about the energy decomposition of an exactly massless pion, particularly in the soft pion limit? What is the microscopic decomposition of the celebrated Gell-Mann-Oakes-Renner (GOR) relation for a pseudo-Goldstone? What is the exact correspondence between the mass decomposition for a heavy quark-antiquark bound state and phenomenological quark potential model? The answers to all these questions in $\mathrm{QCD}_{4}$ appear elusive in the foreseeable future.

In contrast to the notoriously intractable $\mathrm{QCD}_{4}$, twodimensional QCD (hereafter, $\mathrm{QCD}_{2}$ ) in the large- $N$ limit, often referred to as the 't Hooft model [4], is a solvable toy model, which still resembles the realistic QCD in many aspects, such as color confinement, Regge trajectories, chiral symmetry spontaneous breaking, quark-hadron duality, etc. Historically, once an interesting new feature of realistic QCD was established, one would often take the ' $t$ Hooft model as a fruitful laboratory to concretize the results.

In this article, we carry out a systematic investigation on the energy decomposition of a meson in the 't Hooft model, irrespective of whether the meson is stationary or fast moving. We can express each gauge-invariant sector of energy decomposition in terms of the chiral angle and forward (backward)-moving bound-state wave functions. We can answer all the questions raised above. The most nontrivial findings of this article are about meson energy and mass decomposition in the chiral limit and heavy quark 
limit. It is hoped that most features observed in the 't Hooft model may carry over to the decomposition of hadron energy in $\mathrm{QCD}_{4}$.

\section{GAUGE-INVARIANT DECOMPOSITION OF HADRON ENERGY}

The QCD Lagrangian reads

$$
\mathcal{L}_{\mathrm{QCD}}=-\frac{1}{4} F_{\mu \nu}^{a} F^{a \mu \nu}+\bar{\psi}(i D-m) \psi,
$$

where $\psi$ signifies the quark field, $F_{\mu \nu}^{a}$ is the gluon field strength tensor, and $D_{\mu}=\partial_{\mu}-i g_{s} A_{\mu}^{a} T^{a}$ denotes the covariant derivative, with $T^{a}$ denoting the generators of the $S U(N)$ group in the fundamental representation. For simplicity, we will consider only one single flavor of quark throughout this work.

The symmetric energy-momentum tensor of QCD is then

$$
T^{\mu \nu}=\frac{1}{2} \bar{\psi} i \stackrel{\leftrightarrow}{D^{(\mu}} \gamma^{\nu)} \psi+\frac{1}{4} g^{\mu \nu} F^{2}-F^{a \mu \alpha} F_{\alpha}^{a \nu},
$$

where $\stackrel{\leftrightarrow}{D}=\vec{D}-\overleftarrow{D} . D_{\mu}=\partial_{\mu}-i g_{s} A_{\mu}^{a} T^{a}$ denotes the color covariant derivative, and $(\mu, \nu)$ implies the symmetrization of the Lorentz indices $\mu$ and $\nu$.

The $\mathrm{QCD}_{2}$ Hamiltonian can be broken into three pieces,

$$
H=\int d x T^{00}=\mathcal{M}+\mathcal{K}+\mathcal{G},
$$

where three gauge-invariant operators are defined as

$$
\begin{gathered}
\mathcal{M} \equiv \int d x m \bar{\psi} \psi \\
\mathcal{K} \equiv \int d x \frac{1}{2} \bar{\psi} i \stackrel{\leftrightarrow}{D}^{1} \gamma^{1} \psi \\
\mathcal{G} \equiv \int d x\left(\frac{1}{4} F^{2}-F^{a 0 \alpha} F^{a 0}{ }_{\alpha}\right)=\int d x \frac{1}{2}\left(F_{01}^{a}\right)^{2} .
\end{gathered}
$$

For brevity, we will often suppress the superscript 1 in the spatial component of the two-vector $x^{\mu}$; e.g., the symbols $x$ and $x^{1}$ will be used interchangeably, if no confusion arises.

We define the expectation value of an operator $\mathcal{O}$ in a mesonic momentum eigenstate $|P\rangle$ as $\langle\mathcal{O}\rangle \equiv$ $\langle P|\mathcal{O}| P\rangle /\langle P \mid P\rangle$, where the meson state is relativistically normalized according to $\left\langle P \mid P^{\prime}\right\rangle=2 P^{0} 2 \pi \delta\left(P-P^{\prime}\right)$. Sandwiching (3) between any mesonic state carrying momentum $P$, we then expect

$$
P^{0} \equiv \sqrt{M^{2}+P^{2}}=\langle\mathcal{M}\rangle+\langle\mathcal{K}\rangle+\langle\mathcal{G}\rangle,
$$

with $M$ the mass of the meson. For future convenience, we will refer to $\langle\mathcal{M}\rangle,\langle\mathcal{K}\rangle$, and $\langle\mathcal{G}\rangle$ as the quark mass term, quark kinetic energy term, and gluon energy term, respectively.

We remark that, unlike the complication inherent in mass decomposition for $\mathrm{QCD}_{4}$ [1-3], thanks to the superrenormalizablity of $\mathrm{QCD}_{2}$, we need not concern ourselves with the scale-dependence of the matrix elements in (5) nor the trace anomaly associated with (2).

\section{BOUND-STATE SOLUTIONS IN QCD 2}

Let us recapitulate some essential ingredients of the bound-state solutions of the 't Hooft model in equal-time quantization and in axial gauge [5]. First, the large $N$ limit is specified as

$$
N \rightarrow \infty, \quad \lambda \equiv \frac{g_{s}^{2} N}{4 \pi} \text { fixed. }
$$

Of central importance is the so-called mass-gap equation, which can be obtained by diagonalizing the single-particle sector of the dressed-quark Hamiltonian, or equivalently by minimizing the vacuum energy density $[5,6]$,

$p \cos \theta(p)-m \sin \theta(p)=\frac{\lambda}{2} f_{-\infty}^{+\infty} d k \frac{\sin [\theta(p)-\theta(k)]}{(p-k)^{2}}$,

where $f$ denotes the standard principal-value prescription to sweep the infrared singularity as $k \rightarrow p$.

The integral equation (7) can be numerically solved to determine the chiral angle $\theta(p)$ for any given quark mass. Note $\theta(p)$ is an odd function of $p$, and tends to $\pm \pi / 2$ as $p \rightarrow \pm \infty$. It should be stressed that the chiral angle in the massless quark limit still assumes a nontrivial profile, which corresponds to a chiral-asymmetric vacuum carrying a nonzero condensate.

The dressed quark then admits the following dispersive law:

$$
\begin{aligned}
E(p)= & m \cos \theta(p)+p \sin \theta(p) \\
& +\frac{\lambda}{2} f_{-\infty}^{+\infty} d k \frac{\cos [\theta(p)-\theta(k)]}{(p-k)^{2}} .
\end{aligned}
$$

Note that for small quark mass, the dressed quark energy can even become negative for small $p$. This is a harmless and tolerable nuisance, since the colored and gauge-variant entity does not directly correspond to a physical quantity.

With the solutions of $\theta(p)$ and $E(p)$ available, a pair of bound-state equations for the flavor-neutral $q \bar{q}$ mesons were first given by Bars and Green in 1978,

$$
\begin{aligned}
& \left.\left[E(p)+E(P-p) \mp P_{n}^{0}\right)\right] \phi_{ \pm}^{n}(p, P) \\
& =\lambda f_{-\infty}^{+\infty} \frac{d k}{(p-k)^{2}}\left[C(p, k, P) \phi_{ \pm}^{n}(k, P)\right. \\
& \left.\quad-S(p, k, P) \phi_{\mp}^{n}(k, P)\right],
\end{aligned}
$$

where $n$ denotes the principle quantum number of the mesonic family, and $\phi_{ \pm}^{n}$ signify the forward (backward)moving wave functions for the $n$th state. The solutions of (9) determine the eigen energy of the entire mesonic family, $P_{n}^{0}=\sqrt{M_{n}^{2}+P^{2}}$ for a given quark mass and meson spatial momentum $P$, where $M_{n}$ denotes the mass of the $n$th 
excited meson. The trigonometric functions $C$ and $S$ in (9) are defined as [5]

$$
\begin{aligned}
& C(p, k, P)=\cos \frac{\theta(p)-\theta(k)}{2} \cos \frac{\theta(P-p)-\theta(P-k)}{2}, \\
& S(p, k, P)=\sin \frac{\theta(p)-\theta(k)}{2} \sin \frac{\theta(P-p)-\theta(P-k)}{2} .
\end{aligned}
$$

Note that $f$ in (8) and (9) differ from the ordinary principle-value prescription, due to more severe IR divergences encountered there. For a smooth test function $f(y)$, the generalized principle-value prescription is specified as

$$
\begin{aligned}
f \frac{d y}{(x-y)^{2}} f(y)= & \lim _{\epsilon \rightarrow 0}\left[\int^{x-\epsilon} \frac{d y}{(x-y)^{2}} f(y)\right. \\
& \left.+\int_{x+\epsilon} \frac{d y}{(x-y)^{2}} f(y)-2 \frac{f(x)}{\epsilon}\right] .
\end{aligned}
$$

Equation (9) has been numerically solved for stationary [7] and moving [8] mesons, for a variety of quark mass. To simultaneously handel both stationary and moving mesons, we deliberately choose the orthogonality condition of wave functions that differs from [8,9] by a factor of $|P|$ :

$$
\int_{-\infty}^{+\infty} d p\left[\phi_{n}^{+}(p, P) \phi_{m}^{+}(p, P)-\phi_{n}^{-}(p, P) \phi_{m}^{-}(p, P)\right]=\delta_{n m} .
$$

It can be proved that $[5,8]$ with the increasing quark mass/principle quantum number/meson momentum, the backward-moving wave function $\phi_{-}$quickly fades away. In particular, in the $P \rightarrow \infty$ limit, (9) smoothly transitions into the celebrated 't Hooft equation [4],

$$
\frac{m^{2}}{x(1-x)} \phi^{n}(x)-2 \lambda f_{0}^{1} d y \frac{\phi^{n}(y)-\phi^{n}(x)}{(x-y)^{2}}=M_{n}^{2} \phi^{n}(x),
$$

where $\phi_{+}^{n}(p, P)$ in the infinite-momentum frame can be identified with the 't Hooft light-cone wave function (LCWF) $\phi^{n}(x)$, with $x \equiv p / P$ denoting the fraction of the quark momentum with respect to the meson momentum.

\section{ENERGY DECOMPOSITION OF A MOVING MESON}

A particular advantage of the axial gauge $A^{1 a}=0$ is that the 0 -component of the gauge potential becomes a constrained rather than a dynamical variable,

$$
A^{0 a}\left(x^{0}, x\right)=\frac{g_{s}}{2} \int d y|x-y| \psi^{\dagger}\left(x^{0}, y\right) T^{a} \psi\left(x^{0}, y\right),
$$

which is nothing but the instantaneous Coulomb potential in two dimensions. Moreover, when specializing to the axial gauge, $\mathcal{M}$ in (4a) remains intact and the other two in (4) reduce to

$$
\begin{aligned}
\mathcal{K} & =-\int d x \frac{1}{2} \bar{\psi} i \stackrel{\leftrightarrow}{\partial}{ }^{1} \gamma^{1} \psi \\
\mathcal{G} & =\int d x \frac{1}{2}\left(\partial_{1} A^{0}\right)^{2} \\
& =-\frac{g_{s}^{2}}{4} \iint d x d y \psi^{\dagger}(x) T^{a} \psi(x)|x-y| \psi^{\dagger}(y) T^{a} \psi(y) .
\end{aligned}
$$

To compute hadronic matrix elements involving these operators, rather than utilize the diagrammatic BetheSalpeter approach [5], it becomes much more transparent to invoke the operator method, that is, the bosonization technique equipped with Bogoliubov transformation [6]. In fact, these techniques have recently been applied to systematically investigate the partonic quasidistributions in the 't Hooft model [9]. Following the steps expounded in $[6,9]$, and going through some tedious but straightforward algebra, we end up with the following expectation values when sandwiched between the $n$th mesonic state,

$$
\begin{aligned}
\langle\mathcal{M}\rangle_{n}= & m \int_{-\infty}^{\infty} d p[\cos \theta(p)+\cos \theta(\bar{p})]\left[\left(\phi_{+}^{n}(p, P)\right)^{2}+\left(\phi_{-}^{n}(p, P)\right)^{2}\right], \\
\langle\mathcal{K}\rangle_{n}= & \int_{-\infty}^{\infty} d p[p \sin \theta(p)+\bar{p} \sin \theta(\bar{p})]\left[\left(\phi_{+}^{n}(p, P)\right)^{2}+\left(\phi_{-}^{n}(p, P)\right)^{2}\right], \\
\langle\mathcal{G}\rangle_{n}= & \frac{\lambda}{2} \int_{-\infty}^{\infty} d p f_{-\infty}^{\infty} d k \frac{1}{(k-p)^{2}}\{[\cos (\theta(k)-\theta(p)) \\
& +\cos (\theta(\bar{k})-\theta(\bar{p}))]\left[\left(\phi_{+}^{n}(p, P)\right)^{2}+\left(\phi_{-}^{n}(p, P)\right)^{2}\right] \\
& -2 C(p, k, P)\left[\phi_{+}^{n}(k, P) \phi_{+}^{n}(p, P)+\phi_{-}^{n}(k, P) \phi_{-}^{n}(p, P)\right] \\
& \left.+2 S(p, k, P)\left[\phi_{+}^{n}(k, P) \phi_{-}^{n}(p, P)+\phi_{-}^{n}(k, P) \phi_{+}^{n}(p, P)\right]\right\},
\end{aligned}
$$

with $\bar{p} \equiv P-p, \bar{k} \equiv P-k$. With the aid of (9) and orthogonality conditions, one readily verifies that the sum of three pieces in (16) indeed recover $P_{n}^{0}=\sqrt{M_{n}^{2}+P^{2}}$. 
Equation (16) is the key formula of this article, which provides a unified energy decomposition formula, valid for any quark mass and any meson momentum. In the following sections, we will explore the consequence of (16) in several different settings.

We cannot resist remarking that several auxiliary quantities appearing in intermediate stages, such as (8) and (14), are gauge-dependent and plagued with infrared divergences, and by default we have employed the principle-value prescription as a specific IR regulator. In contrast, each individual piece in (16) is gauge invariant as well as IR finite (hence, regulator independent) and can thereby be endowed with some physical significance.

\section{ENERGY DECOMPOSITION IN THE INFINITE-MOMENTUM LIMIT}

It is curious to know how the energy is partitioned among three components with the ever-increasing meson momentum. In general, boosting a bound-state wave function is a highly nontrivial, dynamical, rather than kinematic, operation. Nevertheless, the energy decomposition simplifies substantially when the meson is boosted to the infinitemomentum frame. In the $P \rightarrow \infty$ limit, with $x=p / P$ kept fixed, it is legitimate to replace $\phi_{+}^{n}(p, P)$ by the LCWF $\phi^{n}(x)$, throw away all the $\phi_{-}^{n}$ terms, and take the approximation $\tan \theta(x P) \rightarrow \frac{x P}{m}+\mathcal{O}(1 / P)$ [5,8]. Equation (16) in the $P \rightarrow \infty$ limit then simplifies into

$$
\begin{aligned}
\lim _{P \rightarrow \infty}\langle\mathcal{M}\rangle_{n} & =\frac{m^{2}}{P} \int_{0}^{1} d x \frac{\left(\phi^{n}(x)\right)^{2}}{x(1-x)}, \\
\lim _{P \rightarrow \infty}\langle\mathcal{K}\rangle_{n} & =P-\frac{m^{2}}{2 P} \int_{0}^{1} d x \frac{\left(\phi^{n}(x)\right)^{2}}{x(1-x)}, \\
\lim _{P \rightarrow \infty}\langle\mathcal{G}\rangle_{n} & =\frac{\lambda}{P} \int_{0}^{1} d x f_{0}^{1} d y \frac{\phi^{n}(x)-\phi^{n}(y)}{(x-y)^{2}} \phi^{n}(x) .
\end{aligned}
$$

As an alternative to fathom the behavior of a meson with infinite momentum, one can directly start from the lightcone Hamiltonian. From (2), one can decompose the $\mathrm{QCD}_{2}$ light-cone Hamiltonian into

$$
H_{\mathrm{LC}}=\int d x^{-} T^{+-}=\mathcal{M}_{\mathrm{LC}}+\mathcal{G}_{\mathrm{LC}}
$$

where the light-cone coordinates are defined as $x^{ \pm}=\frac{x^{0} \pm x^{1}}{\sqrt{2}}$. The two gauge-invariant operators are

$\mathcal{M}_{\mathrm{LC}}=\int d x^{-} \frac{m}{2} \bar{\psi} \psi, \quad \mathcal{G}_{\mathrm{LC}}=\frac{1}{2} \int d x^{-} F^{a+-} F_{-+}^{a}$.

Note that there is no counterpart to the quark kinetic energy (4b) in the light-cone decomposition.
One can compute the expectation values of these operators along a similar path which leads to (16). Imposing the light-cone gauge $A^{a+}=0$, and employing the light-cone Hamiltonian operator approach [9], one finally finds

$$
\begin{aligned}
\left\langle\mathcal{M}_{\mathrm{LC}}\right\rangle_{n} & =\frac{P}{P^{+}} \lim _{P \rightarrow \infty}\left[\langle\mathcal{M}\rangle_{n}+\langle\mathcal{K}\rangle_{n}-P\right], \\
\left\langle\mathcal{G}_{\mathrm{LC}}\right\rangle_{n} & =\frac{P}{P^{+}} \lim _{P \rightarrow \infty}\langle\mathcal{G}\rangle_{n} .
\end{aligned}
$$

Following (5), one readily confirms $P_{n}^{-}=\left\langle\mathcal{M}_{\mathrm{LC}}\right\rangle_{n}+$ $\left\langle\mathcal{G}_{\mathrm{LC}}\right\rangle_{n}$, as expected.

\section{NUMERICAL RESULTS}

For numerical calculations, we fix the 't Hooft coupling $\lambda=0.18 / \pi \mathrm{GeV}^{2}$, conforming to the accepted value of the string tension in realistic $\mathrm{QCD}_{4}$ [8]. For simplicity, all masses will be given in units of $\sqrt{2 \lambda}=340 \mathrm{MeV}$. Following [8,9], we consider four different values of quark masses and concentrate on the corresponding four different lowest-lying mesons: chiral (massless) pion $\pi_{\chi}$, physical pion $\pi$, a fictitious strangeonium, and charmonium. For the physical pion and charmonium, the quark mass is tuned such that the realistic masses of $\pi$ and $J / \psi$ are correctly reproduced. The numerical solutions for the chiral angle and Bars-Green equations for these cases were described in detail in [8]. It is then a straightforward exercise to plug these numerical solutions into the integrals in (16) to accomplish the energy decomposition.

In Fig. 1, we plot the energy decompositions against the meson momentum for the aforementioned four types of
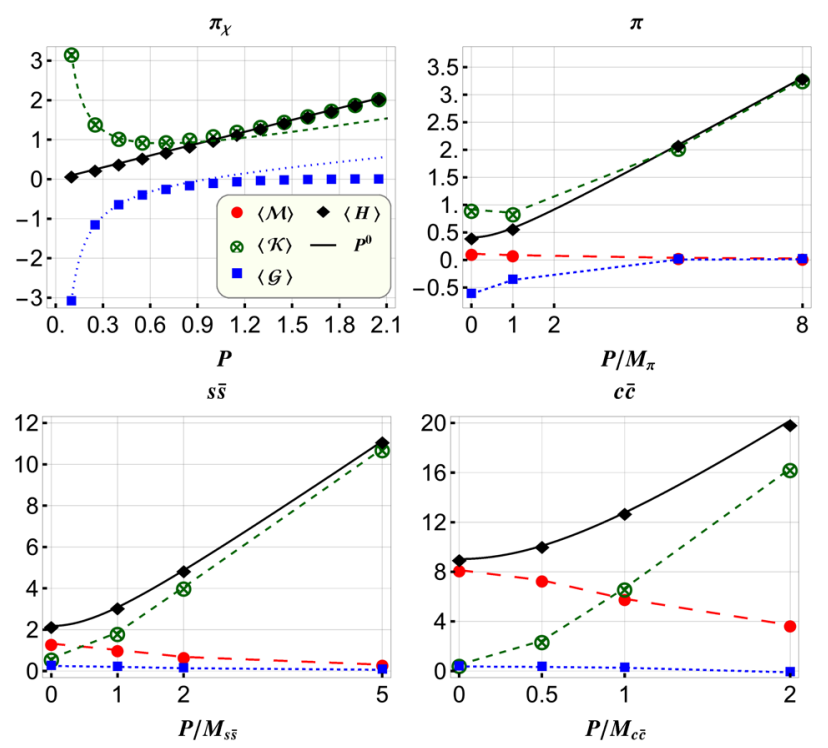

FIG. 1. Numerical predictions for the energy decomposition of four types of ground-state mesons, as a function of the meson momentum. The solid curve denotes $P^{0}=\sqrt{P^{2}+M^{2}}$. 
mesons. Then, we recognize the pattern in the largemomentum limit, as demanded in (17): as a consequence of the Lorentz contraction of the meson's spatial extent, the quark mass term $\langle\mathcal{M}\rangle$ and gluonic term $\langle\mathcal{G}\rangle$ decrease as $1 / P$, but $\langle\mathcal{K}\rangle$, scaling as $P$, completely saturates the meson energy. When the meson momentum gets small, there also arise some peculiar patterns. For heavy quarkonium, unsurprisingly, its rest mass is overwhelmed by the quark mass term and receives a positive contribution from the gluon term. For a lighter meson such as the pion, the gluonic term $\langle\mathcal{G}\rangle$ becomes deeply negative.

\section{ENERGY DECOMPOSITION OF THE SOFT CHIRAL PION AND PHYSICAL PION}

Coleman's theorem states that there cannot arise a Goldstone boson in two spacetime dimensions [10]. Nevertheless, $\mathrm{QCD}_{2}$ in the large $N$ limit appears to be very peculiar in the sense that the ground-state meson for the massless quark is massless, and the theory also admits a nonzero chiral condensate in the chiral limit $[11,12]$. Thus, it is customary to still refer to the massless pseudoscalar meson as the chiral pion $\left(\pi_{\chi}\right)$, a would-be "Goldstone" particle. The Bars-Green wave functions for the chiral pion, $\phi_{ \pm}^{\pi_{\chi}}$, are analytically known in terms of the chiral angle [6], which turn out to be nonanalytic at $P=0$ [8]. Note that, for the soft pion, the backward-moving wave function is not suppressed with respect to the forward-moving one at all, reflecting the nontrivial vacuum structure of the 't Hooft model in the chiral limit [6].

From the upper left panel of Fig. 1, it is amazing to see that $\langle\mathcal{K}\rangle$ and $\langle\mathcal{G}\rangle$ tend to $\pm \infty$ when $P \rightarrow 0$. Fitting the numerical data from $P=10^{-4}$ to $10^{-2}$, these two components are well parametrized by the following form:

$$
\begin{aligned}
\langle\mathcal{K}\rangle_{\pi_{\chi}} & =\frac{0.312}{P}+0.661 P, \\
\langle\mathcal{G}\rangle_{\pi_{\chi}} & =-\frac{0.312}{P}+0.339 P .
\end{aligned}
$$

The divergences cancel when summing up quark kinetic and gluonic pieces. The origin of this singularity can be traced to the huge amplitudes of $\phi_{ \pm}^{\pi_{\chi}}$ in the $P \rightarrow 0$ limit, as a consequence of almost identical profiles of these two wave functions for soft pion. This can be understood from the peculiar minus sign in the normalization condition (12), a characteristic of Bogoliubov transformation [6].

It is certainly interesting to speculate about whether or not a similar singular behavior for the massless pion also arises in $\mathrm{QCD}_{4}$. Unfortunately, it is beyond the contemporary Monte Carlo simulation technique to directly implement the massless quark on the lattice.

We then turn to the mass decomposition of the physical pion, the so-called pseudo-Goldstone boson. Intriguingly, the physical $\pi$ in the 't Hooft model is found to obey the following relation very well,

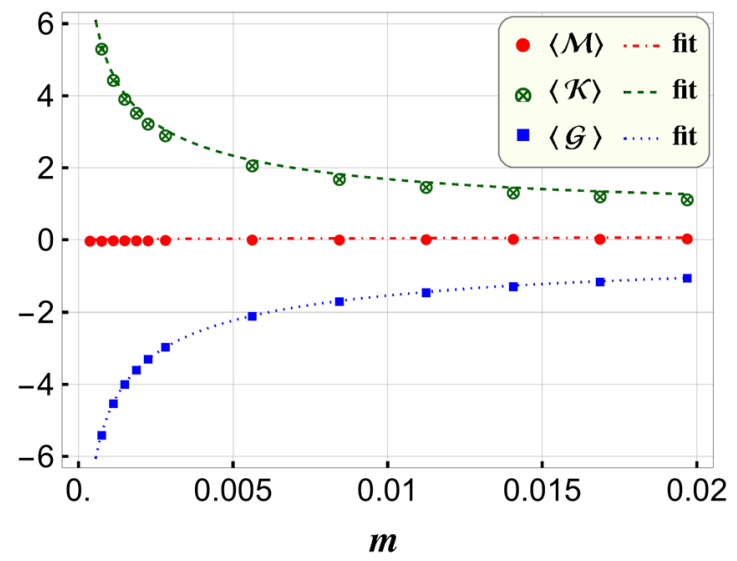

FIG. 2. The light quark mass dependence of $\langle\mathcal{M}\rangle,\langle\mathcal{K}\rangle$ and $\langle\mathcal{G}\rangle$, associated with the mass decomposition of a stationary "pseudoGoldstone" pion.

$$
f_{\pi}^{2} M_{\pi}^{2}=-4 m\langle\Omega|\bar{\psi} \psi| \Omega\rangle,
$$

with $f_{\pi}=\sqrt{N / \pi},\langle\bar{\psi} \psi\rangle=-\sqrt{2 \lambda} N / \sqrt{12}$ in the chiral limit $[11,12]$. Equation (22) is just the two-dimensional counterpart of the celebrated GOR relation [13], directly reflecting the pseudo-Goldstone nature of the pion.

We would like to examine the microscopic origin for the GOR relation in our case. Figure 2 presents the light quark mass dependence of the mass decomposition for the "pseudo Goldstone" boson. In the $m \rightarrow 0$ limit, our numerical results reveal the power-law divergence in the quark kinetic term and gluonic term. Fitting the numerical data in the small $m$ range, the three mass components are found to be well described by the following parametrizations ${ }^{1}$ :

$$
\begin{aligned}
\langle\mathcal{M}\rangle_{\pi} & =0.448 \sqrt{m}+\mathcal{O}(m), \\
\langle\mathcal{K}\rangle_{\pi} & =1.005 m^{-0.288}+6.252 \sqrt{m}-2.719+\mathcal{O}(m), \\
\langle\mathcal{G}\rangle_{\pi} & =-1.005 m^{-0.288}-4.781 \sqrt{m}+2.719+\mathcal{O}(m) .
\end{aligned}
$$

The nonanalytic $m^{-0.288}$ terms, which exhibit some weak logarithmical singularities, cancel upon summing $\langle\mathcal{K}\rangle$ and $\langle\mathcal{G}\rangle$. Each of the components in (23) contains a term $\propto \sqrt{m}$, so all of them contribute to the GOR relation. Note that the sigma term $\langle\bar{\psi} \psi\rangle_{\pi} \propto 1 / \sqrt{m}$ is power divergent in the chiral limit. Summing three pieces in (23), we obtain $M_{\pi}=$ $1.919 \sqrt{m}$, in decent agreement with the GOR relation $M_{\pi}=1.905 \sqrt{m}$, with a relative error: $0.7 \%$.

It is certainly worth speculating whether the decomposition of the GOR relation in $\mathrm{QCD}_{4}$ exhibits a similar

\footnotetext{
${ }^{1}$ To be more general, we may parametrize $\langle\mathcal{M}\rangle_{\pi}$ in the form $c \sqrt{m}+\alpha m^{\beta}$. Nevertheless, the fitting leads to $\beta=0.495 \approx 1 / 2$; thus, these two terms can be merged into a single entity $\propto \sqrt{m}$.
} 
pattern. Considering the steady technological progress, it seems feasible for the lattice simulation to examine the mass decomposition of a physical pion in the real world in the near future.

\section{MASS DECOMPOSITION OF THE STATIONARY HEAVY QUARKONIUM}

For a heavy quark, since $m \gg \sqrt{2 \lambda}$, one may neglect the interaction term in (7) and (8), and, consequently, the chiral angle $\theta(p) \approx \tan ^{-1} \frac{p}{m}$ and $E(p) \approx \sqrt{m^{2}+p^{2}}$. In the rest frame of a heavy quarkonium, the typical velocity of the heavy quark is quite small, so one may further make a nonrelativistic expansion for $\theta(p)$ and $E(p)$. Setting the $\theta$ angle to 0 , approximating $E(p) \approx m+\frac{p^{2}}{2 m}$, and dropping $\phi_{-}$, one can reduce the Bars-Green equations (9) into a single equation,

$$
\frac{p^{2}}{2 \mu} \phi_{+}^{n}(p)-\lambda f_{-\infty}^{\infty} \frac{d k}{(p-k)^{2}} \phi_{+}^{n}(k)=\mathcal{E}_{n} \phi_{+}^{n}(p),
$$

with the reduced mass $\mu=m / 2$ and binding energy $\mathcal{E}_{n} \equiv M_{n}-2 m$. This is nothing but the Schrödinger equation with a linear potential $V(x)=\lambda \pi|x|$ in the momentum space, with $\phi_{+}^{n}(p)$ identified with the corresponding momentum-space Schrödinger wave function. The equivalent Schrödinger equation in the coordinate space reads

$$
\left(\frac{-\partial_{x}^{2}}{2 \mu}+\lambda \pi|x|\right) \psi_{n}(x)=\mathcal{E}_{n} \psi_{n}(x)
$$

with $\psi_{n}(x)$ the coordinate-space wave function for the $n$th excited state. The $\psi_{n}(x) \mathrm{s}$ are known analytically:

$\psi_{n}(x)=N_{n} \times \begin{cases}\operatorname{Ai}\left(\frac{\mu^{1 / 3}\left(2 \pi \lambda x-2 \mathcal{E}_{n}\right)}{(2 \pi \lambda)^{2 / 3}}\right) & x<0, \\ (-1)^{n} \operatorname{Ai}\left(\frac{\mu^{1 / 3}\left(-2 \pi \lambda x-2 \mathcal{E}_{n}\right)}{(2 \pi \lambda)^{2 / 3}}\right) & x>0,\end{cases}$

where Ai is the Airy function, $N_{n}$ is the normalization constant to guarantee $\int d x \psi_{n}^{2}(x)=1$. The energy spectrum of the heavy quarkonium family can be determined through these equations:

$$
\begin{cases}\operatorname{Ai}^{\prime}\left(-\frac{\mathcal{E}_{n}(2 \mu)^{1 / 3}}{(\pi \lambda)^{2 / 3}}\right)=0 & \text { even } n \\ \operatorname{Ai}\left(-\frac{\mathcal{E}_{n}(2 \mu)^{1 / 3}}{(\pi \lambda)^{2 / 3}}\right)=0 & \text { odd } n\end{cases}
$$

Apparently, in the limit of an infinitely heavy quark, the Schrödinger wave function $\psi_{n}(x)$ is linked with the forward-moving Bars-Green wave function in the rest frame, $\phi_{+}^{n}(p, 0)$, through the Fourier transform:

$$
\psi_{n}(x)=\lim _{m \rightarrow \infty} \int_{-\infty}^{\infty} \frac{d p}{2 \pi} \phi_{+}^{n}(p, 0) e^{i p x} .
$$

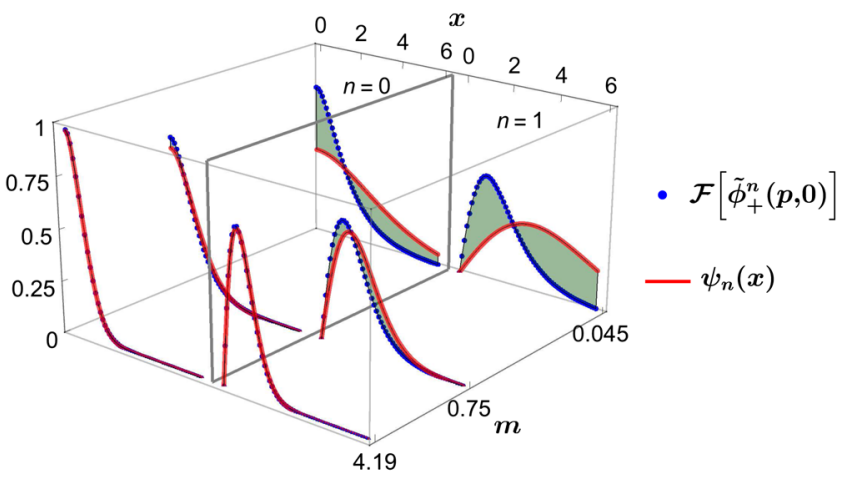

FIG. 3. Comparison between the Fourier-transformed $\phi_{+}^{n}(p, 0)$ and the coordinate-space Schrödinger wave function $\psi_{n}(x)$, with three different quark masses, $m=0.045,0.75,4.19$, corresponding to $u, s, c$, respectively. Both the ground state $n=0$ and the first excited state $n=1$ are plotted. $\mathcal{F}$ denotes the Fourier transform introduced in (28).

We devote Fig. 3 to quantify the difference between $\psi_{n}(x)$, obtained by solving the Schrödinger equation, and the Fourier-transformed $\phi_{+}^{n}(p, 0)$, obtained by solving the Bars-Green equations for a variety of quark masses. One clearly sees that, when $m$ reaches the charm quark mass, these two wave functions converge satisfactorily.

In a similar vein, expanding (16) in the heavy quark limit, we obtain

$$
\begin{aligned}
\lim _{m \rightarrow \infty}\langle\mathcal{M}\rangle_{n} & =2 m-\int_{-\infty}^{\infty} d p \frac{p^{2}}{2 \mu} \phi_{+}^{n}(p)^{2}, \\
\lim _{m \rightarrow \infty}\langle\mathcal{K}\rangle_{n} & =\int_{-\infty}^{\infty} d p \frac{p^{2}}{\mu} \phi_{+}^{n}(p)^{2}, \\
\lim _{m \rightarrow \infty}\langle\mathcal{G}\rangle_{n} & =\lambda \int_{-\infty}^{\infty} d p f_{-\infty}^{\infty} d k \frac{\phi_{+}^{n}(p)^{2}-\phi_{+}^{n}(k) \phi_{+}^{n}(p)}{(p-k)^{2}} .
\end{aligned}
$$

We, thus, can identify the expectation values of the kinetic and potential energy of the $n$th state in quantum mechanics with the field-theoretical matrix elements in (29),

$$
\begin{aligned}
\langle K\rangle_{n} & \equiv\left\langle n\left|\frac{\hat{p}^{2}}{2 \mu}\right| n\right\rangle=\int d x \psi_{n}(x) \frac{-\partial_{x}^{2}}{2 \mu} \psi_{n}(x) \\
& =\lim _{m \rightarrow \infty}\left[\langle\mathcal{M}\rangle_{n}+\langle\mathcal{K}\rangle_{n}\right]-2 m, \\
\langle V\rangle_{n} & \equiv\langle n|\lambda \pi| x|| n\rangle=\int d x \psi_{n}(x) \lambda \pi|x| \psi_{n}(x) \\
& =\lim _{m \rightarrow \infty}\langle\mathcal{G}\rangle_{n},
\end{aligned}
$$

where $\langle V\rangle_{n}=2\langle K\rangle_{n}$ in line with the virial theorem. From Fig. 4, one sees that the kinetic energy obtained in single-particle quantum mechanics already agrees well with $\langle\mathcal{M}\rangle+\langle\mathcal{K}\rangle-2 m$ for $c \bar{c}$. Nevertheless, to have a decent 


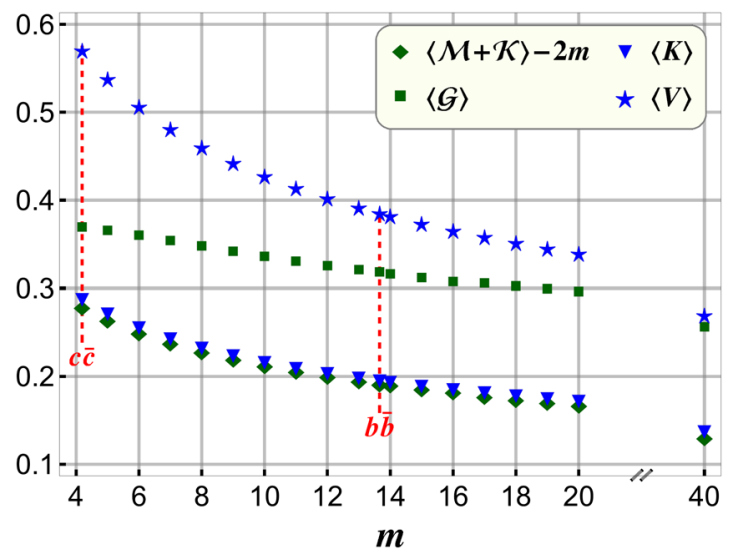

FIG. 4. Comparison between the kinetic and potential energy in quantum mechanics and those field-theoretical mass components in (30). Only the ground state quarkonium $(n=0)$ is considered.

agreement between potential energy and $\langle\mathcal{G}\rangle$, the quark appears to be at least 3 times heavier than the $b$ quark. It is interesting to note that, although the Schrödinger wave function and the forward-moving Bars-Green wave function already coincide in shape for the $c \bar{c}$ family, $\langle\mathcal{G}\rangle$ and $\langle V\rangle$ exhibit a much slower convergence tendency. This may indicate that, for a modestly heavy quark, the true profile of the chiral angle may still play an indispensable role in the mass breakup of quarkonium.

\section{SUMMARY}

We have studied the gauge-invariant energy decomposition of a flavor-neutral meson within the framework of the 't Hooft model. The energy of a meson can be decomposed into three gauge-invariant components, i.e., quark mass term, quark kinetic term, and gluonic term, each of which can be expressed in terms of the chiral angle and the forward (backward)-moving bound-state wave functions. In the chiral limit, we find an amazing feature- that the quark kinetic energy and gluonic energy for a massless "Goldstone pion" diverge as $\pm 1 / P$ as the meson momentum $P \rightarrow 0$. For small yet nonzero quark mass, we observe that both the quark kinetic and gluonic energy of the "pseudo-Goldstone" pion possess nonanalytic power-law dependence of $m$ together with $\sqrt{m}$. We are able to conduct a microscopic decomposition for the GOR relation in the two-dimensional QCD. In the heavy quark limit, we illustrate how the field-theoretical decomposition of the binding energy for a heavy quarkonium can recover the familiar nonrelativistic quantum mechanics. It is hoped that these interesting features observed in $\mathrm{QCD}_{2}$ can offer some inspiration and serve as a prototype for our understanding of energy decomposition of hadrons in realistic $\mathrm{QCD}_{4}$.

\section{ACKNOWLEDGMENTS}

The work of Y. J. and R. Y. is supported in part by the National Natural Science Foundation of China under Grants No. 11475188 and No. 11621131001 (CRC110 by DFG and NSFC). The work of X.-N.X. is supported by the Deutsche Forschungsgemeinschaft (Sino-German CRC 110).
[1] X. D. Ji, Phys. Rev. Lett. 74, 1071 (1995).

[2] X. D. Ji, Phys. Rev. D 52, 271 (1995).

[3] Y. B. Yang, Y. Chen, T. Draper, M. Gong, K.-F. Liu, Z. Liu, and J.-P. Ma, Phys. Rev. D 91, 074516 (2015).

[4] G. 't Hooft, Nucl. Phys. B75, 461 (1974).

[5] I. Bars and M. B. Green, Phys. Rev. D 17, 537 (1978).

[6] Y. S. Kalashnikova and A. V. Nefediev, Usp. Fiz. Nauk 172, 377 (2002) [Phys. Usp. 45, 347 (2002)].

[7] M. Li, L. Wilets, and M. C. Birse, J. Phys. G 13, 915 (1987).
[8] Y. Jia, S. Liang, L. Li, and X. Xiong, J. High Energy Phys. 11 (2017) 151.

[9] Y. Jia, S. Liang, X. Xiong, and R. Yu, Phys. Rev. D 98, 054011 (2018).

[10] S. R. Coleman, Commun. Math. Phys. 31, 259 (1973).

[11] A. R. Zhitnitsky, Phys. Lett. 165B, 405 (1985);

[12] M. Li, Phys. Rev. D 34, 3888 (1986).

[13] M. Gell-Mann, R. J. Oakes, and B. Renner, Phys. Rev. 175, 2195 (1968). 